08

\title{
Собственные частоты изгибных колебаний углеродных нанотрубок
}

\author{
() С.В. Дмитриев, ${ }^{1}$ И.Р. Сунагатова, ${ }^{2}$ М.А. Ильгамов, ${ }^{2,3,4}$ И.С. Павлов ${ }^{1}$ \\ ${ }^{1}$ Федеральный исследовательский центр Институт прикладной физики РАН, \\ 603024 Нижний Новгород, Россия \\ ${ }^{2}$ Башкирский государственный университет, \\ 450076 Уфра, Россия \\ ${ }^{3}$ Институт машиноведения им. А.А. Благонравова РАН, \\ 101990 Москва, Россия \\ ${ }^{4}$ Институт механики им. Р.Р. Мавлютова УФИЦ РАН, \\ 450054 Уфра, Россия \\ e-mail: sunagatova66@gmail.com
}

Поступило в Редакцию 28 апреля 2021 г.

В окончательной редакции 16 июня 2021 г.

Принято к публикации 29 июня 2021 г.

С использованием молекулярно-динамической модели с сокращенным числом степеней свободы рассчитаны собственные частоты изгибных колебаний углеродных нанотрубок (УНТ) различного диаметра в условиях плоского деформированного состояния. Показано, что теория тонких цилиндрических оболочек дает высокую точность в оценке частот малоамплитудных собственных колебаний даже для УНТ относительно малого диаметра. Показано, что с ростом амплитуды частота собственных колебаний уменьшается, что согласуется с литературными данными. Полученные результаты необходимы для дизайна терагерцовых резонаторов на основе УНТ и высокоточных наносенсоров массы и силы, основанных на эффекте электромеханической связи, который проявляют УНТ.

Ключевые слова: углеродные нанотрубки, изгибные колебания, молекулярная динамика, теория оболочек, наносенсоры.

DOI: 10.21883/JTF.2021.11.51536.127-21

\section{Введение}

Поскольку $s p^{2}$-углеродные материалы, такие как графеновые наноленты и углеродные нанотрубки (УНТ), имеют высокие прочность и жесткость, а также хорошо проводят электрический ток, естественно возникает идея использования электромеханической связи для создания наносенсоров $[1,2]$ и нано- и микроэлектромеханических систем (NEMS и MEMS) различного назначения [3]. B таких наноустройствах через углеродные структуры пропускают постоянный или переменный ток, по изменению которого можно судить о действии внешних сил, изменении температуры или химического состава окружающей среды. Наибольшую чувствительность подобные сенсоры демонстрируют вблизи резонансных частот механических колебаний рассматриваемых материалов. Механические резонаторы можно использовать в качестве инерционных весов для обнаружения малой массы вещества (порядка ag) путем измерения сдвигов частоты колебаний [4-9], а также для измерения весьма малых сил (порядка $\mathrm{nN}$ ) $[1,10,11]$. В этой связи становится актуальной задача поиска простых способов оценки собственных частот колебаний $s p^{2}$-углеродных структур в зависимости от их геометрических параметров.

Для решения этой задачи используются различные модели. Несмотря на наноскопический размер углеродных структур, многими авторами было показано, что континуальные модели механики сплошной среды оказываются применимыми для анализа их колебательных спектров [12-21]. В настоящей работе оцениваются пределы применимости теории тонких упругих оболочек к анализу изгибных собственных колебаний УНТ. В качестве более точного метода расчета используется молекулярная динамика, учитывающая дискретное строение УНТ и межатомные потенциалы Савина [22], точность которых при описании механических свойств $s p^{2}$-углеродных структур была показана в ряде работ [23]. Для расчетов применяется модель цепи, движущейся на плоскости [24,25], которая позволяет для некоторого класса задач многократно уменьшить число рассматриваемых степеней свободы при сохранении высокой точности моделирования механических свойств $s p^{2}$-углеродных материалов. Модель цепи была адаптирована для анализа УНТ в работе [26] и успешно использована для описания механических свойств пучков УНТ при поперечном сжатии [27-30].

\section{1. Компьютерная модель}

В настоящей работе рассматриваются УНТ в условиях плоского деформированного состояния, когда форма поперечного сечения УНТ полностью определяет ее деформированное состояние, при этом деформация растяжения/сжатия вдоль оси УНТ равна нулю. Для определенности рассматриваются УНТ хиральности зиг- 


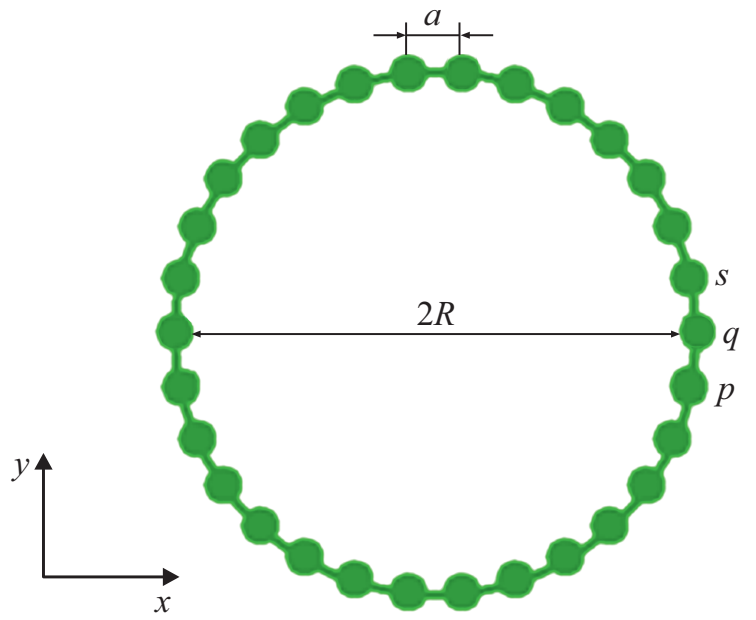

Рис. 1. Поперечное сечение УНТ хиральности зигзаг $(K, 0)$ на плоскости $(x, y)$. Показан случай $K=15$, при этом поперечное сечение характеризуется $N=2 K=30$ атомами углерода.

заг $(K, 0)$ различного диаметра, определяемого параметром $K$, т. е. направление зигзаг графена направлено по окружности УНТ, а направление кресло вдоль оси УНТ [19]. На рис. 1 схематически показана расчетная ячейка и определены геометрические параметры: $a-$ расстояние между атомами углерода в стенке УНТ в проекции на плоскость $(x, y)$ и $R$ - радиус УНТ. Атомы имеют две степени свободы, двигаясь в плоскости $(x, y)$. Каждый узел представляет собой жесткий ряд атомов углерода, ориентированный перпендикулярно плоскости $(x, y)$. Поперечное сечение УНТ представлено $N=2 K$ атомами углерода. Рассмотрены случаи $N=\{18,30,42,54,66,78\}$. Численные значения геометрических параметров следующие. Равновесное межатомное расстояние в стенке УНТ равно $r_{0}=1.418 \AA$, расстояние между ближайшими атомами углерода в проекции на плоскость $(x, y)$ равно $a=r_{0} \sqrt{3 / 2}=1.228 \AA$, радиус УНТ рассчитывается как $R=a /[2 \sin (\pi / N)]$. Заметим, что радиус УНТ в нашем исследовании относительно невелик и при отсутствии внешних сил они могут иметь только круговую устойчивую конфигурацию.

Положения атомов на плоскости $(x, y)$ определяются компонентами радиус-вектора $\mathbf{r}$, при этом общее число степеней свободы равно $2 N$. Рассматриваемая УНТ находится в вакууме. В молекулярно-динамическом моделировании единицами измерения времени, энергии и расстояния являются $\mathrm{ps}, \mathrm{eV}$ и $\AA$ соответственно. В этих единицах масса атома углерода равна $M=12 \cdot 1.0364 \cdot 10^{-4} \mathrm{eV} \cdot \mathrm{ps}^{2} / \AA^{2}$.

Движение атомов УНТ описывается гамильтонианом (полной энергией)

$$
H=T+U_{B}+U_{A},
$$

представляющим сумму кинетической энергии, энергии валентных связей и валентных углов (их выражения приводятся в [29], а также ниже для удобства читателя).
На рис. 1 индексы $p, q$ и $s$ нумеруют ближайшие атомы в стенке УНТ. Тогда энергии в правой части уравнения (1) определяются следующим образом.

Кинетическая энергия

$$
T=\frac{M}{2} \sum_{q=1}^{N}\left|\dot{\mathbf{r}}_{q}\right|^{2},
$$

где $\dot{\mathbf{r}}_{q}$ - вектор скорости атома с номером $q=1, \ldots, N$, точкой обозначено дифференцирование по времени.

Энергия валентных связей определяется гармоническим потенциалом

$$
U_{B}=\frac{k}{2} \sum_{q=1}^{N}\left(\left|\mathbf{r}_{q}-\mathbf{r}_{s}\right|-a\right)^{2},
$$

который суммируется по связям, соединяющим ближайшие атомы (рис. 1), и число таких связей равно числу атомов $N$. Продольная жесткость графена хорошо воспроизводится при $k=405 \mathrm{~N} / \mathrm{m}=25.278 \mathrm{eV} / \AA^{2}$.

Энергия валентных углов задается ангармоническим потенциалом

$$
\begin{gathered}
U A=\epsilon \sum_{q=1}^{N}\left[1+\cos \left(\theta_{p q s}\right)\right], \\
\cos \left(\theta_{p q s}\right)=\frac{\left(r_{q}-r_{p}, r_{s}-r_{q}\right)}{\left|r_{q}-r_{p}\right|\left|r_{s}-r_{q}\right|},
\end{gathered}
$$

который является суммированием по валентным углам, образованным атомами $p, q$ и $s$ (рис. 1), и число таких углов равно числу атомов $N$. Изгибная жесткость графена хорошо воспроизводится при $\epsilon=3.50 \mathrm{eV}$.

Уравнения движения, следующие из гамильтониана (1)-(4), решались численно методом Штормера шестого порядка точности [31].

Атомы УНТ занумерованы индексом $q=1, \ldots, N$ против часовой стрелки, начиная с атома, расположенного на положительной оси $x$. Таким образом, координаты атомов равны $x=R \cos [2 \pi(q-1) / N]+x_{0}$, $y=R \sin [2 \pi(q-1) / N]+y_{0}$, где $x_{0}, y_{0}-$ координаты центра УНТ.

Для возбуждения в УНТ изгибных колебаний атомам с координатами $(x, y)$ задавался вектор начальных перемещений с компонентами

$$
\begin{aligned}
& \Delta x=A \frac{x-x_{0}}{R} \cos \left(\frac{2 \pi n(q-1)}{N}\right), \\
& \Delta y=A \frac{y-y_{0}}{R} \cos \left(\frac{2 \pi n(q-1)}{N}\right) .
\end{aligned}
$$

Здесь $(\Delta x, \Delta y)$ - компоненты вектора начального смещения атома с координатами $x, y ; n-$ номер гармоники; $q-$ номер атома; $A-$ амплитуда гармоники. Задание начальных перемещений атомов, согласно (5), создает в УНТ прогиб с $n$ волнами, как показано на 

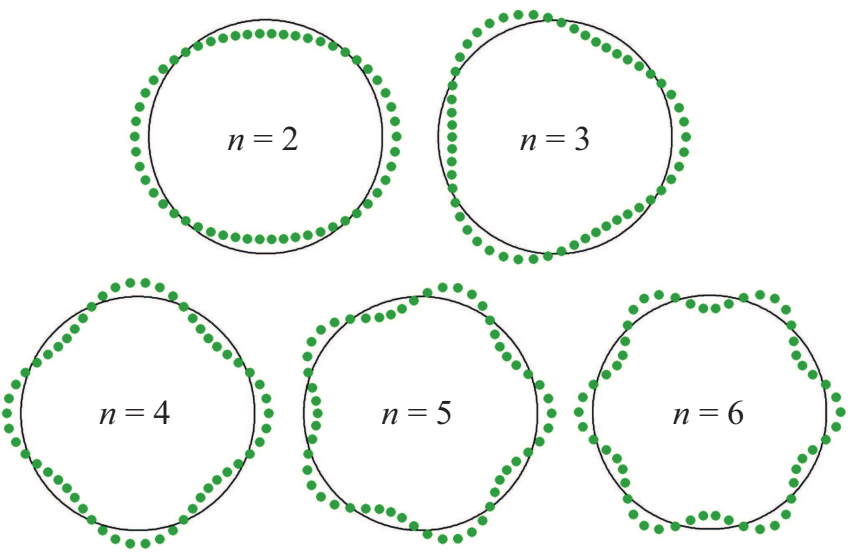

Рис. 2. Первые пять гармоник изгибных колебаний УНТ, соответствующих номерам $n=2,3, \ldots, 6$ в выражении (5). Показано на примере УНТ (27.0), для которой $N=54$ и радиус $R=9.47 \AA$. В этом случае при $n=6$ на длину волны приходится 9 атомов.

рис. 2 для случая УНТ с $N=54(R=9.47 \AA)$. Нами рассматривались случаи $n=2,3, \ldots, 6$. Начальные скорости всех атомов равнялись нулю. В результате таких начальных условий в УНТ возбуждались свободные колебания, соответствующие выбранной гармонике. Частота колебаний определялась по зависимости радиальных перемещений атомов как функций времени. При определении частот малоамплитудных колебаний мы полагали $A=10^{-3} \AA$. При изучении нелинейных колебаний брались бо́льшие значения $A$.

Теория тонких упругих оболочек дает следующее выражение для собственных частот изгибных колебаний цилиндрической оболочки [32]:

$$
v_{n}=\frac{\xi n\left(n^{2}-1\right)}{2 \pi R^{2} \sqrt{n^{2}+1}},
$$

где $n$ - номер гармоники, $R$ - радиус цилиндрической оболочки и $\xi-$ коэффициент, учитывающий инерцию и цилиндрическую жесткость стенки оболочки. Отметим, что номер гармоники $n$ в теории тонких упругих оболочек имеет физический смысл модуля проекции углового момента кванта колебаний (фонона) на ось УНТ. Из уравнения (6) находим периоды собственных колебаний:

$$
T_{n}=\frac{2 \pi R^{2} \sqrt{n^{2}+1}}{\xi n\left(n^{2}-1\right)}
$$

В разд. 2 периоды и частоты пяти первых гармоник изгибных колебаний УНТ будут рассчитаны с использованием молекулярно-динамической модели, описанной выше. Коэффициент $\xi$ будет найден подстановкой в формулу (7) радиуса УНТ $R$, номера гармоники $n$ и периода колебаний соответствующей гармоники $T_{n}$, найденной из молекулярно-динамических расчетов. Можно отметить, что континуальная теория должна давать наилучший результат для оценки коэффициента $\xi$ при использовании длинноволновой гармоники $(n=2)$, наименее чувствительной к дискретности УНТ.

\section{2. Численные результаты}

С использованием модели цепи, описанной в разд. 1, были рассчитаны частоты и периоды малоамплитудных изгибных колебаний УНТ различного радиуса для первых пяти гармоник, показанных на рис. 2. Результаты сравниваются с аналитической оценкой (6). Также анализируется эффект нелинейности при значительных амплитудах колебаний.

Напомним, что радиус УНТ определяется числом атомов углерода $N$ в ее поперечном сечении. Частоты малоамплитудных колебаний как функции номера гармоники $n$ представлены на рис. 3 для $N=18,30,42$, 54, 66 и 78 различными символами согласно вставке. Хорошо видно, что частота колебаний растет с номером гармоники $n$ быстрее, чем по линейному закону и быстро уменьшается с ростом $N$.

Используем численно найденные периоды колебаний $n$-ой гармоники для определения значения коэффициента $\xi$, входящего в (7), для каждого радиуса УНТ. Например, для случая $N=78$, находим радиус УНТ $R=13.68 \AA$ А. Подставляя численно найденное значение периода колебаний $T_{2}=7.571 \mathrm{ps}$ для $n=2$ в уравнение (7), определяем $\xi=58.12 \AA^{2} / \mathrm{ps}$. В таблице сведены результаты аналогичных расчетов для других значений $N$ и соответствующих радиусов $R$.

Анализируя зависимость $\xi$ от числа $N$ и радиуса УНТ при $n=2$ (см. таблицу), замечаем, что $\xi$ возрастает замедленно с ростом $R$, достигая насыщения при $R>13.68 \AA$ на уровне $\xi=58.12 \AA^{2} / \mathrm{ps}$. Это означает, что для УНТ радиуса $R>13.68 \AA$ размерный эффект перестает проявляться. Поэтому теория упругих оболочек может использоваться для оценки частот изгибных колебаний таких УНТ.

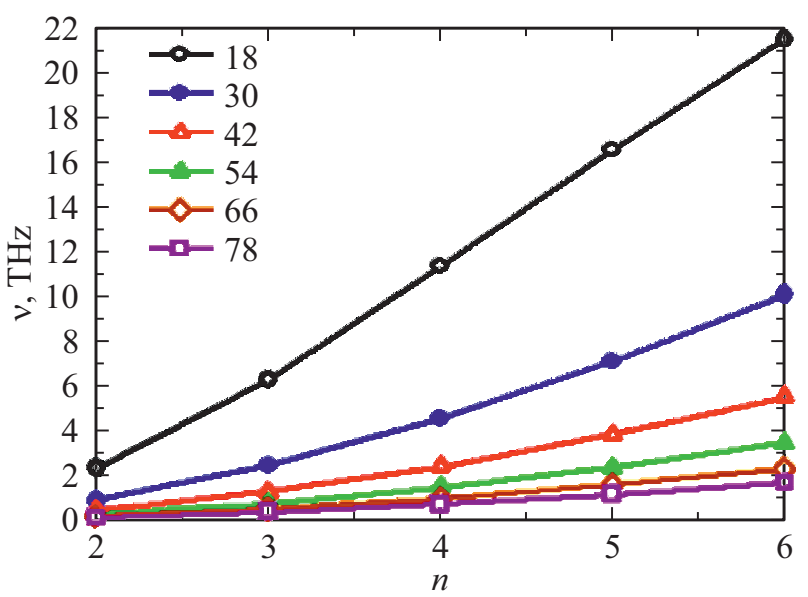

Рис. 3. Частоты первых пяти гармоник собственных изгибных колебаний УНТ для различных значений $N$ от 18 до 78 с шагом 12. 

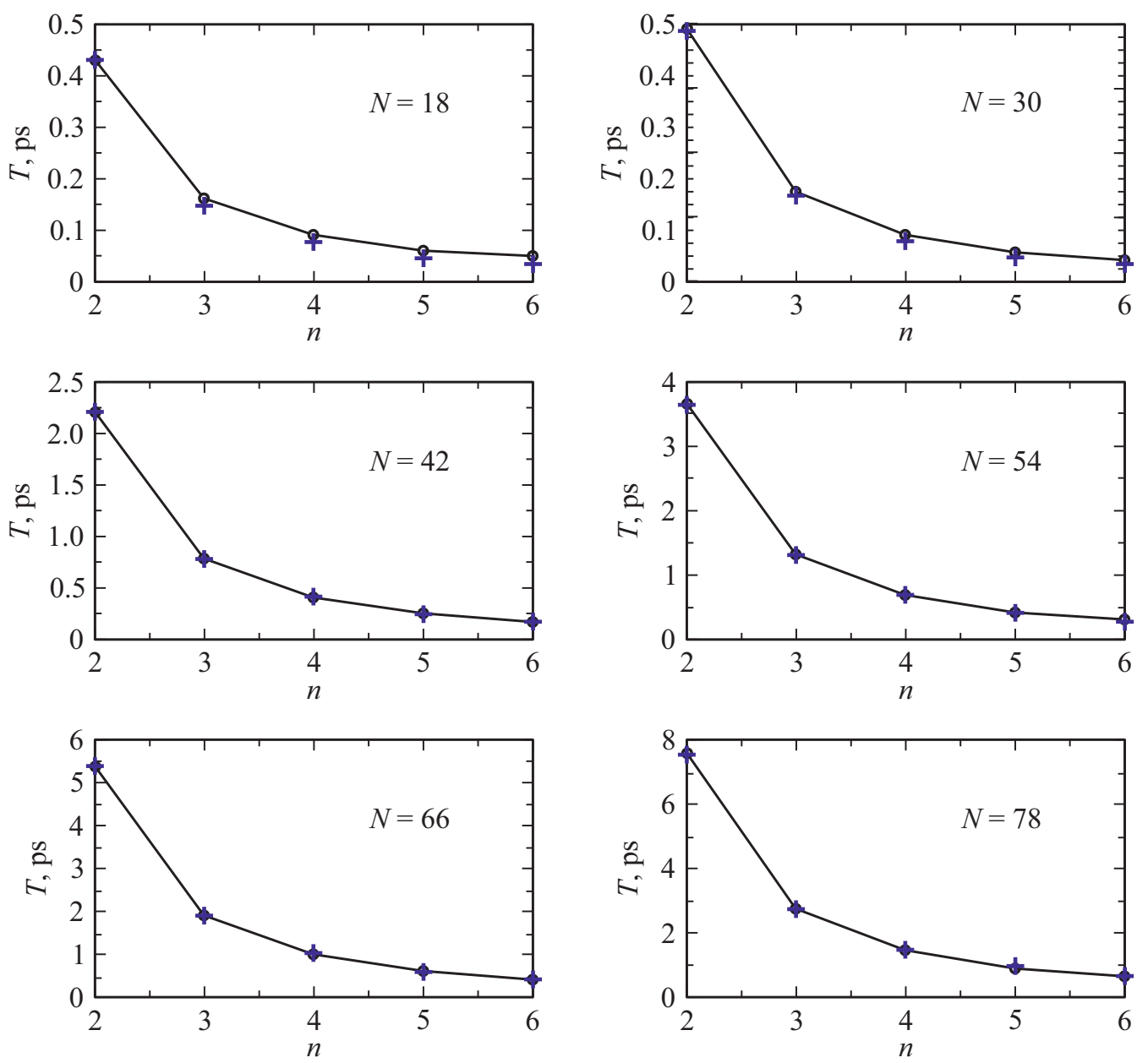

Рис. 4. Период малоамплитудных изгибных колебаний УНТ для первых пяти гармоник, занумерованных индексом $n=2,3, \ldots, 6$, согласно численным результатам (пустые символы) и предсказанию по формуле (7) (крестики) при определении коэффициента $\xi$ по гармонике $n=2$. Число атомов $N$ в поперечном сечении УНТ указано на каждой панели.

Радиус УНТ $R$ и коэффициент $\xi$, входящий в выражения (6) и (7), для различных значений числа атомов углерода в поперечном сечении УНТ $N$ и для различных гармоник от $n=2$ до 6

\begin{tabular}{c|c|c|c|c|c|c}
\hline \multirow{2}{*}{$N$} & \multirow{2}{*}{$R, \AA$} & \multicolumn{5}{|c}{$\xi, \AA^{2} / \mathrm{ps}$} \\
\cline { 3 - 7 } & & $n=2$ & $n=3$ & $n=4$ & $n=5$ & $n=6$ \\
\hline 18 & 3.17 & 54.88 & 52.38 & 49.58 & 44.58 & 39.48 \\
30 & 5.27 & 56.24 & 55.80 & 54.58 & 52.86 & 51.04 \\
42 & 7.37 & 57.46 & 57.04 & 56.29 & 55.45 & 54.48 \\
54 & 9.47 & 57.67 & 57.37 & 56.98 & 56.64 & 55.97 \\
66 & 11.58 & 58.02 & 57.50 & 57.41 & 57.03 & 56.71 \\
78 & 13.68 & 58.12 & 57.58 & 57.63 & 57.31 & 57.14
\end{tabular}

Отметим, что в качестве параметра, характеризующего степень дискретности колебательной моды, удобно взять отношение $N / n$, т.е. число узлов цепной модели, приходящейся на одну длину волны колебательной моды. Наименьшее из исследованных нами значение этого соотношения равно $N / n=18 / 6=3$, а наибольшее $N / n=78 / 2=39$. Континуальная теория, согласно нашим оценкам, может быть использована для $N / n>78 / 6=13$.

Зависимость коэффициента $\xi$ от числа атомов $N$ и номера гармоники $n$ в соответствии с данными таблицы может быть представлена приближенной формулой (в $\left.\AA^{2} / \mathrm{ps}\right)$ :

$$
\xi=61\left(1-e^{N / 6}\right)\left(1-e^{-6 / n}\right)
$$

для $18 \leq N \leq 78,2 \leq n \leq 6$,

$$
\xi=58 \quad \text { для } \quad N>78, \quad 2 \leq n \leq 6 .
$$

На рис. 4 сопоставлены численные результаты (пустые символы) и предсказание по формуле (7) (сплошная кривая) для периодов первых пяти гармоник изгибных колебаний УНТ с различным числом атомов $N$ (указано на каждой панели). Значение параметра $\xi$ для каждого $N$ бралось из таблицы для $n=2$. Все кривые проходят через точку, соответствующую $n=2$, поскольку она использовалась для определения параметра $\xi$. 

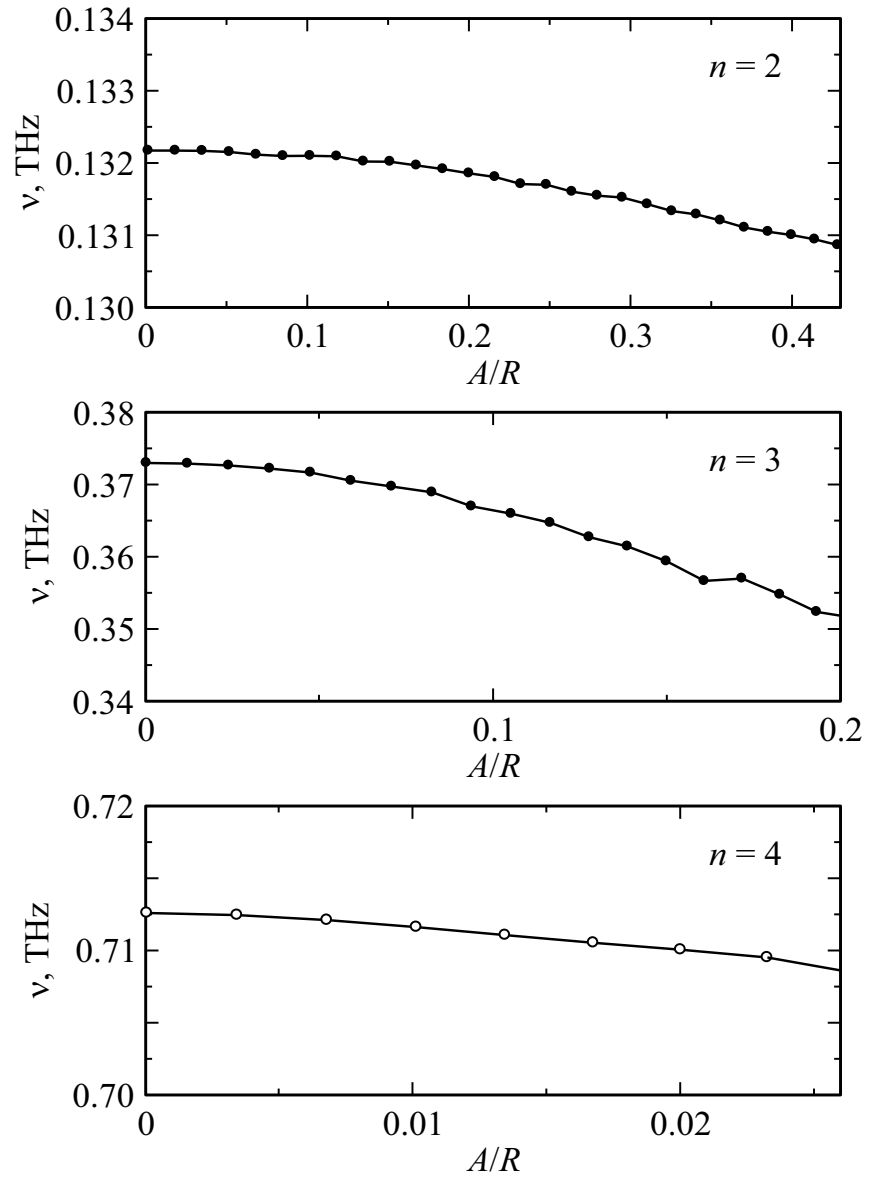

Рис. 5. Зависимость частоты колебаний от амплитуды для гармоник $n=2,3$ и 4. Результаты для УНТ радиуса $R=15.25 \AA$ $(N=78)$. Амплитуда колебаний $A$ нормирована на радиус УНТ.

Отклонение кривой от численных значений возрастает с ростом $n$, поскольку дискретность УНТ становится более выраженной для коротковолновых мод колебаний. Наибольшая погрешность наблюдается для $n=6$. Для $N=18$ и $n=6$ максимальная погрешность достигает $32 \%$, что не удивительно, поскольку в этом случае в пределах длины волны колебаний находится всего три атома углерода. С ростом $N$ максимальная погрешность быстро падает и для $N=78$ она составляет всего $1.1 \%$.

Наконец, рассмотрим влияние амплитуды колебаний на частоту для УНТ радиуса $R=13.68 \AA \quad(N=78)$. Для первых трех гармоник $(n=2,3$ и 4) результаты молекулярно-динамического моделирования показаны на рис. 5 с указанием номера гармоники на каждой панели. Амплитуда колебаний нормирована на радиус УНТ. Отметим, что частота колебаний уменьшается с ростом амплитуды, однако степень нелинейности колебаний невелика. Для гармоники $n=2$ падение частоты во всем исследованном интервале амплитуды составляет около $1 \%$, для гармоники $n=3$ частота падает на $9 \%$, а для $n=4$ на $0.6 \%$. Отметим, что для гармоники $n=3$ не удалось рассмотреть амплитуды свыше $A / R=0.027$, поскольку при более высоких амплитудах энергия от рассматриваемой гармоники довольно быстро переходит к другим гармоникам. По этой же причине (быстрый обмен энергией между гармониками при увеличении амплитуды колебаний) не удалось проанализировать зависимость частоты от амплитуды для гармоник $n=5$ и 6 .

\section{Заключение}

Сопоставлены результаты молекулярно-динамического расчета частот собственных изгибных колебаний УНТ различного диаметра с предсказанием теории тонких упругих оболочек. Установлено, что для УНТ радиуса больше $13.68 \AA$ коэффициент $\xi$ в аналитическом выражении частот колебаний (6) равен $\xi=58.12 \AA^{2} / \mathrm{ps}$. Для УНТ радиуса $R>13.68 \AA$ размерный эффект перестает проявляться, и теория оболочек может использоваться с высокой точностью для оценки частот изгибных колебаний. Об этом также свидетельствует малость погрешности в оценке частот старших гармоник при радиусе УНТ $R>13.68 \AA$. Так, для $N=78$ погрешность в оценке частоты гармоники $n=6$ составляет всего $1.12 \%$.

Полученные результаты для УНТ достаточно большого радиуса хорошо согласуются с литературными данными. Отметим, что коэффициент $\xi$, фигурирующий в выражениях (6) и (7), также входит в дисперсионное соотношение изгибных колебаний графена: $\omega(k)=\xi k^{2}$. В работе [16] этот коэффициент обозначен через $\gamma$ и для него дана оценка $\gamma=6 \cdot 10^{-3} \mathrm{~cm}^{2} / \mathrm{s}=60 \AA^{2} / \mathrm{ps}$ со ссылкой на первопринципные расчеты [17]. Эта оценка хорошо согласуется со значением $\xi=58.1 \AA^{2} / \mathrm{ps}$, взятым из таблицы для моды $n=2$ и максимального радиуса УНТ. Оценка $\xi=65.6 \AA^{2} / \mathrm{ps}$, основанная на континуальной теории оболочек, дается в [19]. Существует и экспериментальная оценка этого коэффициента, равная $\xi=67.3 \AA^{2} / \mathrm{ps}$ [18], близкая к теоретическому значению, указанному в [33], $\xi=68.2 \AA^{2} /$ ps. Отметим, что расхождение экспериментальных оценок констант упругости графена и УНТ составляет порядка 10\%, что обусловлено не только погрешностью эксперимента, но и зависимостью упругих характеристик от способа синтеза материалов, наличия дефектов, химической модификации и других факторов [34]. Разброс в оценке констант упругости приводит к разбросу в оценке колебательных спектров, поэтому отличие экспериментальных и теоретических результатов в $10 \%$ следует считать удовлетворительным.

С уменьшением радиуса УНТ точность континуальной теории снижается. Аппроксимирующие выражения (8) могут использоваться для расчета частот колебаний УНТ малого радиуса.

C ростом амплитуды колебаний за счет геометрической и физической нелинейности УНТ наблюдается снижение частоты собственных изгибных колебаний (рис. 5). Мягкий тип нелинейности собственных изгибных колебаний УНТ также отмечался в [15]. 
Таким образом, для оценки нескольких первых собственных частот изгибных колебаний УНТ радиуса свыше $13.68 \AA$ можно пользоваться простой формулой (6) с коэффициентом $\xi=58.12 \AA^{2} / \mathrm{ps}$. Полученные результаты полезны для создания терагерцовых резонаторов и наносенсоров малых масс и сил, основанных на использовании резонансных частот колебаний УНТ, проявляющих эффект электромеханической связи.

Отметим, что в работе предполагалось, что УНТ находятся в вакууме. В случае если УНТ взаимодействуют со средой, их колебательный спектр должен рассчитываться с учетом этого обстоятельства [20,32].

\section{Благодарности}

Работа выполнена при финансовой поддержке Российского научного фонда, грант № 21-19-00813.

\section{Конфликт интересов}

Авторы заявляют, что у них нет конфликта интересов.

\section{Список литературы}

[1] C.-Y. Li, T.-W. Chou. Nanotechnology, 15, 1493 (2004). https://doi.org/10.1088/0957-4484/15/11/021

[2] J.-X. Shi, X.-W. Lei, T. Natsuki. Sensors, 21, 1907 (2021). https://doi.org/10.3390/s21051907

[3] H.B. Khaniki, M.H. Ghayesh, M. Amabili. Int. J. Nonlinear Mech., 129, 103658 (2021). https://doi.org/10.1016/j.ijnonlinmec.2020.103658

[4] G. Abadal, Z.J. Davis, N. Barniol, et al. Nanotechnology, 12, 100 (2001). https://doi.org/10.1088/0957-4484/12/2/305

[5] C. Li, T.-W. Chou. Appl. Phys. Lett., 84, 5246 (2004). https://doi.org/10.1063/1.1764933

[6] T. Natsuki, N. Matsuyama, Q.-Q. Ni. Appl. Phys. A, 120, 1309 (2015).

[7] A. Bouchaala, A.H. Nayfeh, M.I. Younis. J. Dyn. Syst. Meas. Control, 138, 091002 (2016).

[8] S.S. Ghaffari, S. Ceballes, A. Abdelkefi. Smart Mater. Struct., 28, 074003 (2019).

[9] S.S. Ghaffari, S. Ceballes, A. Abdelkefi. Nonlinear Dyn., 100, 1013 (2020).

[10] T. Natsuki, K. Urakami. Electronics, 8, 1082 (2019).

[11] F. Menacer, A. Kadr, Z. Dibi. Int. J. Autom. Comput., 17, 279 (2018).

[12] X.-W. Lei, Q.-Q. Ni, J.-X. Shi, T. Natsuki. Nanoscale Res. Lett., 6, 492 (2011).

[13] S. Fazelzadeh, E. Ghavanloo. Compos. Struct., 94, 1016 (2012).

[14] R. Ansari, H. Rouhi, S. Sahmani. J. Vib. Control, 20, 670 (2012).

[15] K. Avramov. Int. J. Non-Linear Mech., 107, 149 (2018).

[16] P.S.V. Goupalov. Phys. Rev. B, 71, 085420 (2005).

[17] D. Sánchez-Portal, E. Artacho, J.M. Soler, A. Rubio, P. Ordejón Phys. Rev. B, 59, 12678 (1999). https://doi.org/10.1103/PhysRevB.59.12678

[18] G.D. Mahan. Phys. Rev. B, 65, 235402 (2002).

[19] S.S. Savinskii, V.A. Petrovskii. Phys. Solid State, 44, 1802 (2002).
[20] Y.M. Sirenko, M.A. Stroscio, K.W. Kim. Phys. Rev. E, 53, 1003 (1996).

[21] B.I. Yakobson, C.J. Brabec, J. Bernholc. Phys. Rev. Lett., 76, 2511 (1996).

[22] A.V. Savin, Y.S. Kivshar, B. Hu. Phys. Rev. B, 82, 195422 (2010). https://doi.org/10.1103/PhysRevB.82.195422

[23] I. Evazzade, I.P. Lobzenko, E.A. Korznikova, I.A. Ovid'Ko, M.R. Roknabadi, S.V. Dmitriev. Phys. Rev. B, 95, 035423 (2017). https://doi.org/10.1103/PhysRevB.95.035423

[24] A.V. Savin, E.A. Korznikova, S.V. Dmitriev. Phys. Rev. B, 102, 245432 (2020). https://doi.org/10.1103/PhysRevB.102.245432

[25] A.V. Savin, E.A. Korznikova, A.M. Krivtsov, S.V. Dmitriev. Eur. J. Mech. A-Solid., 80, 103920 (2020). https://doi.org/10.1016/j.euromechsol.2019.103920

[26] E.A. Korznikova, L.K. Rysaeva, A.V. Savin, E.G. Soboleva, E.G. Ekomasov, M.A. Ilgamov, S.V. Dmitriev. Materials, 12, 3951 (2019). https://doi.org/10.3390/ma12233951

[27] L.K. Rysaeva, D.V. Bachurin, R.T. Murzaev, D.U. Abdullina, E.A. Korznikova, R.R. Mulyukov, S.V. Dmitriev. Facta Universitatis, Series: Mechan. Eng., 18, 525 (2020). https://doi.org/10.22190/FUME201005043R

[28] L.K. Rysaeva, E.A. Korznikova, R.T. Murzaev, D.U. Abdullina, A.A. Kudreyko, J.A. Baimova, D.S. Lisovenko, S.V. Dmitriev. Facta Universitatis, Series: Mechan. Eng., 18, 1 (2020). https://doi.org/10.22190/FUME200128011R

[29] D.U. Abdullina, E.A. Korznikova, V.I. Dubinko, D.V. Laptev, A.A. Kudreyko, E.G. Soboleva, S.V. Dmitriev, K. Zhou. Computation, 8, 27, (2020). https://doi.org/10.3390/COMPUTATION8020027

[30] S.V. Dmitriev, A.S. Semenov, A.V. Savin, M.A. Ilgamov, D.V. Bachurin. J. Micromechan. Molecular Phys., 2050010 (2021). https://doi.org/10.1142/S2424913020500101

[31] N.S. Bakhvalov. Numerical Methods: Analysis, Algebra, Ordinary Differential Equations (MIR Publishers, M., 1977)

[32] М.А. Ильгамов. Колебания упругих оболочек, содержсащих жидкость и газ (Наука, М., 1969)

[33] A.M. Rao, J. Chen, E. Richter, U. Schlecht, P.C. Eklund, R.C. Haddon, U.D. Venkateswaran, Y.-K. Kwon, D. Tománek. Phys. Rev. Lett., 86, 3895 (2001).

[34] R. Al-Jishi, G. Dresselhaus. Phys. Rev. B, 26, 4514 (1982). 